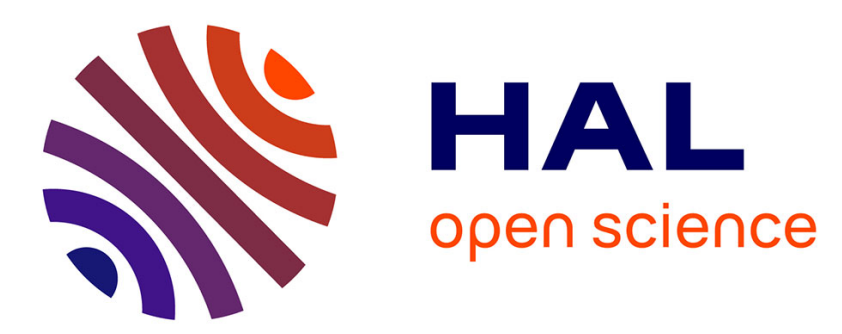

\title{
Time-Delay Identification in a Chaotic Semiconductor Laser With Optical Feedback: A Dynamical Point of View
}

Damien Rontani, A. Locquet, Marc Sciamanna, David S Citrin, Silvia Ortin

\section{- To cite this version:}

Damien Rontani, A. Locquet, Marc Sciamanna, David S Citrin, Silvia Ortin. Time-Delay Identification in a Chaotic Semiconductor Laser With Optical Feedback: A Dynamical Point of View. IEEE Journal of Quantum Electronics, 2009, 45 (7), pp.879-1891. 10.1109/JQE.2009.2013116 . hal-03079791

\section{HAL Id: hal-03079791 \\ https://hal.science/hal-03079791}

Submitted on 17 Dec 2020

HAL is a multi-disciplinary open access archive for the deposit and dissemination of scientific research documents, whether they are published or not. The documents may come from teaching and research institutions in France or abroad, or from public or private research centers.
L'archive ouverte pluridisciplinaire HAL, est destinée au dépôt et à la diffusion de documents scientifiques de niveau recherche, publiés ou non, émanant des établissements d'enseignement et de recherche français ou étrangers, des laboratoires publics ou privés. 


\title{
Time-delay identification in the chaotic output of a semiconductor laser with optical feedback: a dynamical point of view
}

\author{
D. Rontani, A. Locquet, M. Sciamanna, D. S. Citrin, and S. Ortin
}

\begin{abstract}
A critical issue in optical chaos-based communications is the possibility to identify the parameters of the chaotic emitter, and hence to break the security. In this paper, we study theoretically the identification of a chaotic emitter that consists of a semiconductor laser with an optical feedback. The identification of a critical security parameter, the external-cavity-round trip time (the time delay in the laser dynamics), is performed using both the auto-correlation function and delayed mutual information methods applied to the chaotic time-series. The influence on the time-delay identification of the experimentally tunable parameters, the feedback rate, the pumping current and the time-delay value, is carefully studied. We show that difficult time-delay-identification scenarios strongly depend on the timescales of the system dynamics as it undergoes a route to chaos, in particular on how close is the relaxation oscillation period from the external-cavity-roundtrip time.
\end{abstract}

Index Terms-Semiconductor laser, optical feedback, nonlinear dynamics, time-delay identification

\section{INTRODUCTION}

$\mathbf{S}$ EMICONDUCTOR lasers with an external cavity (ECSL) have been considered as rich sources of optical chaos, with well-known chaotic regimes such as the so-called coherence collapse [1] and low-frequency fluctuations (LFF) regimes [2]. Chaotic regimes from ECSL have received considerable attention since they constitute key elements of optical secure chaotic communications [3]. Indeed, the addition of an external cavity introduces an infinite numbers of degree of freedom through the time-delay in the dynamical representation of

D. Rontani is with the Ecole Supérieure d'Electricité (Supélec), Laboratoire Matériaux Optiques, Photonique et Systèmes (LMOPS), Centre National de la Recherche Scientifique (CNRS), F-57070 Metz, France and with and with the Unité Mixte Internationale UMI 2958 GeorgiaTech-CNRS, 2-3 rue Marconi F-57070 Metz, France. He is also with the School of Electrical and Computer Engineering, Georgia Institute of Technology, Atlanta, GA, 30332. (e-mail: damien.rontani@supelec.fr)

A. Locquet is with the Unite Mixte Internationale UMI 2958 GeorgiaTech-CNRS, 2-3 rue Marconi F-57070 Metz, France (e-mail: alocquet@georgiatech-metz.fr)

M. Sciamanna is with the Ecole Supérieure d'Electricité (Supélec), Laboratoire Matériaux Optiques, Photonique et Systèmes (LMOPS), Centre National de la Recherche Scientifique (CNRS), F-57070 Metz, France and with the Unité Mixte Internationale UMI 2958 GeorgiaTech-CNRS, 2-3 rue Marconi F-57070 Metz, France (e-mail: marc.sciamanna@supelec.fr)

D. S. Citrin with the School of Electrical and Computer Engineering, Georgia Institute of Technology, Atlanta, GA, 30332 and with the Unité Mixte Internationale UMI 2958 GeorgiaTech-CNRS, 2-3 rue Marconi F-57070 Metz. (e-mail: david.citrin@ece.gatech.edu)

S. Ortin is with the Instituto de Física de Cantabria, Consejo Superior de Investigaciones Científicas (CSIC)-Universidad de Cantabria, Santander E39005, Spain (e-mail: ortin@ifca.unican.es)

Manuscript received xxxx, 2008; revised xxxx, 2008. the semiconductor laser, leading to the generation of highdimensional chaos [4]. The unpredictable and noisy appearance of the signals emitted by hyper-chaotic optical systems has been used to enhance security and privacy in communications: A chaotic carrier conceals and transmits an information bearing message, which is decrypted by synchronization at the receiver end (a physical copy of the chaotic emitter) [3], [5]-[8]. The security of such a chaos-based communication scheme relies mainly on the difficulty to identify the emitter parameters, and on the sensitivity of synchronization to parameter mismatch [9]. Therefore, it is of particular importance to study the capability of an eavesdropper to retrieve information about the parameters of a particular chaotic generator from its time series transmitted through the communication channel.

In delayed hyper-chaotic systems, the security assumption is based on the computational complexity to reconstruct a high-dimensional attractor from the time series. However, the knowledge of the time delay allows for the projection of the high-dimensional attractor onto a reduced-dimensional phase space, which makes the system vulnerable to lowcomputational-complexity identification techniques [10]. It is thus crucial that the delay not be easily identifiable in a cryptosystem. Until recently, ECSLs with a single optical feedback were considered as weakly secure systems in terms of time-delay identification, such that the use of several external cavities has been suggested [12]. However, we have shown recently that a simple ECSL with a single optical feedback could, with a careful choice of parameters, hide its time-delay signature when standard estimators are employed [13]. Interestingly the region of laser and feedback parameters where such a difficult time-delay identification occurs does not necessarily correspond to the situation where the chaos complexity (dimension and entropy) is higher [4]. Indeed in laser diodes with optical feedback high-dimensional chaos is typically found where the optical feedback strength is large, but then the time-delay parameter is easily retrieved from the analysis of the chaotic output using straightforward techniques [13]. Time-delay identification should therefore be considered as an additional argument to appreciate the level of security of chaos-based identification, besides chaos complexity and robustness of chaos synchronization.

In this paper, we extend our earlier work on time-delay identification in laser diode with optical feedback. Identification is processed using both conventional technique based on autocorrelation function (ACF) and more sophisticated technique such as delayed mutual information (DMI). We 
analyze further the range of laser and feedback parameters that lead to difficult parameter identification and link these bad identification scenarios to the laser dynamics as it undergoes a bifurcation cascade to optical chaos. More specifically we show that the time-scales of the laser dynamics in its route to chaos influence the time-delay identification and therefore also the security of such a chaotic emitter. A careful tuning of the external cavity round-trip time with respect to the intrinsic laser relaxation oscillation frequency leads to situations where the time-delay signature is lost in the laser chaotic output. Finally we discuss the robustness of our results using other signal processing techniques applied to the laser chaotic timeseries.

The paper is organized as followed. Section II presents the model and specificities of the various identification techniques used to retrieve the time-delay value. Section III details the influence of the different operational parameters (the feedback rate, the time-delay and the pumping current) on the identification. Section IV gives a dynamical interpretation of the identification scenarios observed, based on the studies of the frequencies that appear in the ECSL dynamics as it undergoes a route to chaos. Section V discusses the influence of key internal parameters that modify the ECSL model and the possible application of our results to chaos-based communications. Finally, we summarize our main conclusions in section VI.

\section{THEORETICAL FRAMEWORK}

\section{A. Rate equation model}

In this work, we consider a single-mode semiconductor laser with optical feedback modelled by the Lang-Kobayashi rate equations [16]. This model has been successful in reproducing dynamical behaviors experimentally observed in ECSLs. The rate equations are

$$
\begin{aligned}
\frac{d E(t)}{d t}= & \frac{1}{2}(1+i \alpha)\left(G_{N,|E|^{2}}-\frac{1}{\tau_{p}}\right) E(t), \\
& +\gamma e^{i \omega_{0} \tau} E(t-\tau)+F(t) \\
\frac{d N}{d t}= & p J_{t h}-\frac{N}{\tau_{s}}-G_{N,|E|^{2}}|E(t)|^{2},
\end{aligned}
$$

where $E(t)=|E(t)| e^{i \phi(t)}$ is the slowly varying complex electric field, $N$ is the average carrier density in the active region, $\alpha$ is the linewidth-enhancement factor that describes the amplitude phase coupling, $G_{N,|E|^{2}}=\frac{G_{N}\left(N-N_{0}\right)}{1+\varepsilon|E|^{2}}$ is the optical gain where $\varepsilon$ is the saturation coefficient, $N_{0}$ is the carrier density at transparency, $\omega_{0}$ is the angular frequency of the solitary laser, $\gamma$ is the feedback rate, $\tau_{p}$ is the photon lifetime, $\tau_{s}$ is the carrier lifetime, $J_{t h}$ is the threshold current, $p$ is the pumping factor, and $\tau$ is the delay corresponding to the round-trip time of light in the external cavity. The force $F(t)=F_{D}(t)+F_{L}(t)$ added in the field equation models the spontaneous emission occurring in the semiconductor laser. It is composed of a deterministic part $F_{D}(t)=2 \beta \frac{N(t)}{|E(t)|} e^{i \phi(t)}$ and a stochastic part $F_{L}(t)=\sqrt{2 \beta N(t)} \zeta(t)$ which corresponds to a Langevin term where $\zeta=\zeta_{E}(t)+i \zeta_{\phi}(t)$ is a complex Gaussian white-noise term with zero mean and correlation: $\left\langle\zeta_{E}(t) \zeta_{\phi}^{*}\left(t^{\prime}\right)\right\rangle=\delta_{E \phi} \delta\left(t-t^{\prime}\right) . \beta$ is the spontaneous-emission rate. The relaxation-oscillation period is an intrinsic damping time of the free-running laser and is defined by $\tau_{R O}=\left(\frac{1}{\tau_{p} \tau_{s}}(\mu-1)-\frac{\mu^{2}}{4 \tau_{s}^{2}}\right)^{-1 / 2}$ with $\mu=$ $G_{N} \tau_{s} \tau_{p}\left(p J_{t h}-\frac{N_{0}}{\tau_{s}}\right)$. We consider the following parameter values: $\alpha=5, \omega_{0} \tau=0 \mathrm{rad}, \tau_{p}=2 \mathrm{ps}, \tau_{s}=2 \mathrm{~ns}$, $G_{N}=7.5 \times 10^{-13} \mathrm{~m}^{3} \mathrm{~s}^{-1}, N_{0}=3 \times 10^{24} \mathrm{~m}^{-3}, J_{t h}=$ $1.83 \times 10^{33} \mathrm{~m}^{-3} \mathrm{~s}^{-1}, \varepsilon=2.5 \times 10^{-23} \mathrm{~m}^{3}$, and $\beta=10^{3} \mathrm{~s}^{-1}$.

\section{B. Time-delay estimator and time-delay signature}

The time delay is a key parameter of delay systems. Its estimation is of particular importance with regard to computationally efficient identification of all the parameters of the nonlinear system under consideration. Standard techniques, such as the ACF and the DMI, can be used to identify the time-delay.

1) The autocorrelation function (ACF): If a random process $X(t)$ is ergodic and wide-sense-stationary, then the ACF is defined by

$$
\Gamma(\theta)=\frac{1}{\hat{\sigma}_{X}^{2}}\left\langle\left(x(t)-\hat{\mu}_{X}\right)\left(x(t+\theta)-\hat{\mu}_{X}\right)\right\rangle,
$$

where $x(t)$ and $x(t+\theta)$ are sampled from the random process $X(t), \hat{\mu}_{X}=\langle x(t)\rangle$, and $\hat{\sigma}_{X}=\left\langle\left(x(t)-\hat{\mu}_{X}\right)^{2}\right\rangle^{1 / 2}$ with $\langle\cdot\rangle$ denoting time average. The ACF measures for a given value $\theta$, the tendency of the cloud of points $(x(t), x(t+\theta))$ to be aligned along a straight line, and thus measures a linear relationship between $x(t)$ and $x(t+\theta)$.

2) The delayed mutual information (DMI): The mutual information, $H$, is a quantity originally used in information theory [21]. Given two continuous variables $X$ and $Y$ with joint probability density function, $f_{X Y}(x, y)$ and marginal probability density functions $f_{X}(x)$ and $f_{Y}(y)$, the mutual information of $X$ and $Y$ is defined as

$$
H(X, Y)=\mathbb{E}\left(\ln \left(\frac{f_{X Y}(x, y)}{f_{X}(x) f_{Y}(y)}\right)\right),
$$

where $\mathbb{E}(\cdot)$ is the expectancy operator, the two variables $X$ and $Y$ are obtained by sampling the random process $X(t)$ at two times $t$ and $t+\theta$, and such process is assumed to be stationary and ergodic. The probability density functions, $f_{X Y}$, $f_{X}$, and $f_{Y}$, will be estimated by their respective histogram $\hat{f}_{X Y}, \hat{f}_{X}$, and $\hat{f}_{Y}$ computed from the time series and leading to the approximate mutual information estimator, also called delayed mutual information (DMI),

$$
H(\theta)=\mathbb{E}\left(\ln \left(\frac{\hat{f}_{X(t) X(t+\theta)}(x, y)}{\hat{f}_{X(t)}(x) \hat{f}_{X(t+\theta)}(y)}\right)\right) .
$$

The mutual information corresponds intuitively to the quantity of information that the two random variables $X(t)$ and $X(t+\theta)$ are sharing. In time-delay systems, the presence of a delayed feedback term induces a nonlocal time dependence in the time evolution of its state variables. The integral definition of the estimators under consideration allows for the 
detection of nonlocal time dependencies, linear for the ACF and nonlinear for the DMI. Therefore, if a particular timescale, such as a time delay, is present in a given time series, it should manifest itself in the estimator through a local extremum. This signature of the time scale, depending on its sign, will be referred to as peak or valley later on in this article. The time location of a peak (respectively valley) will be considered as a possible estimation of the time delay.

\section{IDENTIFICATION OF TIME DELAY IN LANG-KOBAYASHI EQUATIONS}

In this section, the security in terms of time-delay identification is performed by analyzing the intensity time series of a chaotic ECSL. The intensity is $I(t)=|E(t)|^{2}$. The timedelay signatures in the ACF and the DMI are analyzed with the evolution of the following operational parameters of the ECSL: the feedback rate $\gamma$, the external-cavity-roundtrip time $\tau$, and the pumping factor $p$. Throughout this study, the spontaneousemission rate $\beta$ is taken equal to zero.

\section{A. Influence of the feedback rate}

The feedback rate controls the optical power reinjected in the laser cavity. As a consequence, it drives the contribution of the delayed intensity $I(t-\tau)$ in the time-evolution of $I(t)$. We thus expect that the stronger the feedback rate, the more important the information shared between $I(t-\tau)$ and $I(t)$ will be.

Figure 1 analyzes a first case of time-delay identification, for increasing values of the feedback rate $\gamma$. Large values of the feedback rate produce chaotic time series with a clear signature of the time delay in the estimators: a significant peak is present in both the ACF [Fig. 1(b4)] and the DMI [Fig. 1(c4)], at a time corresponding to the time-delay. Similar results have been obtained experimentally, from the analysis of the autocorrelation function [11]. The progressive decrease in feedback rate produces the following: first, a decrease of the peak's amplitude close to the time delay location and its multiples, in both the ACF and DMI [Fig. 1(b3) and c(3)] until it reaches a minimum value [Fig. 1(b2) and (c2)]; second, a qualitative change of behavior appears. For relatively weak feedback rates, numerous peaks and valleys, separated by $\tau_{R O} / 2$ from each other, appear and are amplified in the vicinity of the origin $(\theta=0)$ and the time delay $(\theta \approx \tau)$ [Fig. 1(b1) and (c1)]. They obscure the time-delay identification. A quantitative description of the feedback-rate influence in terms of time location and amplitude of the time-delay signature is given in Fig. 2 for both the ACF and the DMI. Figure 2 is obtained by considering the most significant peak (or valley) in a vicinity $W(\tau)$ of the time delay which is defined by $\max _{\theta \in W(\tau)}|\Gamma(\theta)|$ for the ACF and $\max _{\theta \in W(\tau)}|H(\theta)|$ for the DMI. In Fig. 2, the curves with triangle markers are drawn for the same parameters as the ones of Fig. 1. The curves confirm the tendency already observed in Fig. 1: as the feedback rate is decreased, the amplitude starts to diminish until a global minimum value is reached, and then it increases [Fig. 2(a1)-(a2)]. The decreasing region of the curve corresponds to weak chaotic regime; therefore, the
ACF and DMI may still find a structural relationship within the intensity time-series $I(t)$. Then for a sufficiently strong feedback rate, the chaotic regime becomes well established and most of the timescale signatures are weakened due to equivalent driving action of the intrinsic nonlinearity of the ECSL and the linearly introduced feedback term. However, if $\gamma$ is further increased, then due to this linear introduction of the delayed term, $\gamma e^{i \omega_{0} \tau} E(t-\tau) e^{i \phi(t-\tau)}$, in the rate equations, the influence of the time delay is enhanced. The decrease in feedback rate also influences the time location of the largest peak at a time close to the time-delay; see Figs. 2(b1)-(b2). For small feedback rates, the largest autocorrelation or mutual information is obtained for a time close to a high order multiple of $\tau_{R O} / 2$, when analyzing a small time window in the vicinity of the time delay $W(\tau)$. Then the identification gives a value of time which is quite shifted from the expected value $(\tau)$. However, as the feedback rate increases, the greatest autocorrelation and mutual information values are achieved for a time closer to the time-delay. This explains the monotonic decreasing behavior of the time shift identification curve in Fig. 2 (b1)-(b2).

In summary this first analysis shows that when the feedback rate is relatively weak, the chaotic output of the laser diode shows only small evidence of the time-delay signature, both in the ACF or the DMI. The largest contribution to the ACF or DMI, in a time window around the time-delay, is in fact closer to a high multiple of the relaxation oscillation period $\tau_{R O}$ and this blurs the good identification of the expected value $\tau$.

Figure 2 also shows the influence of the pumping factor $p$, which controls the injection current $J=p J_{t h}$ as well as the value of the relaxation oscillation period $\tau_{R O}$. When increasing $p$, chaotic dynamics are observed for larger values of the feedback rate $\gamma$ but similar conclusions hold for the identification of the time-delay. The amplitude of the highest peak (or valley) in the ACF and DMI in the vicinity of $\tau$ exhibits a minimum value when the feedback rate increases, and the feedback rate corresponding to this minimum increases with the increase of $p$. Moreover the time value obtained from the ACF or DMI identification techniques is shifted from the expected value $(\tau)$ when the feedback rate is small but gets closer to $\tau$ as the feedback rate increases. The maximum time shift is however smaller when $p$ increases, hence progressively leading to a better identification of $\tau$.

\section{$B$. Influence of the time delay relatively to the relaxation- oscillation period}

In this subsection, we study how the value $\tau$ affects the time-delay identification based on the ACF and DMI. This influence is analyzed relatively to the value of the relaxationoscillation period $\tau_{R O}$, the other significant ECSL timescale in terms of identification. This line of reasoning is supported by the results of Section III.A, where the two timescales coexist and $\tau_{R O}$ proves to have a blurring effect on the time-delay signature [Fig. 1(b1)-(c1)]. Here, we will show that $\tau_{R O}$ may have an even stronger effect. If it is taken sufficiently close to $\tau$ and provided that the pumping current is small $(p \approx 1$, which leads to relatively large values of $\tau_{R O}$ ), then the time- 

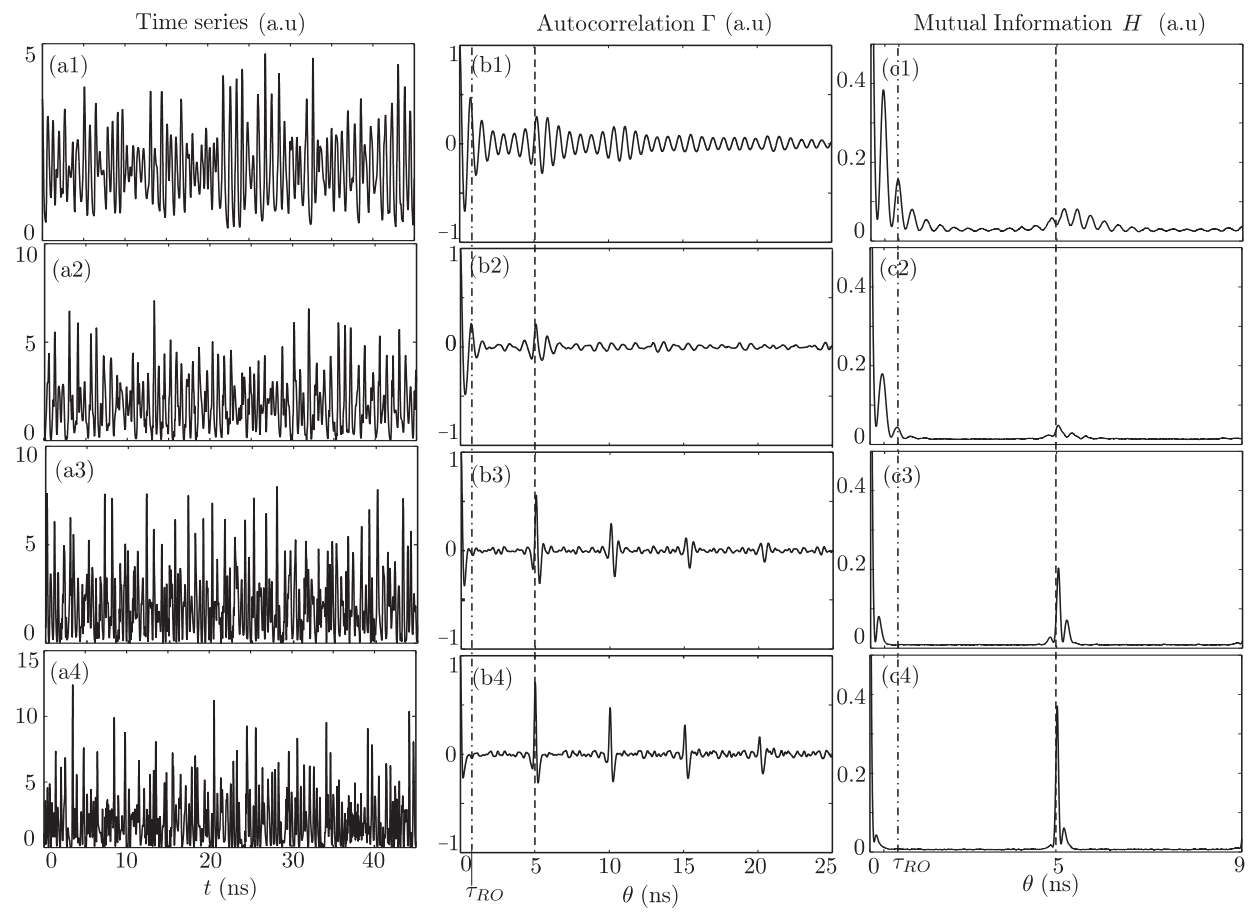

Fig. 1. ECSL intensity time series (1st column), Autocorrelation function (2nd column) and mutual information (3rd column) for increasing value of the feedback rate $\gamma=2 \mathrm{GHz}$ (1st row), $\gamma=5 \mathrm{GHz}$ (2nd row), $\gamma=10 \mathrm{GHz}$ (3rd row), $\gamma=15 \mathrm{GHz}$ (4th row) with a time-delay value $\tau=5 \mathrm{~ns}$ and $\tau_{R O}=0.75 \mathrm{~ns}$. The vertical dotted-dashed and dashed lines give the time location of $\tau_{R O}$ and $\tau$, respectively.
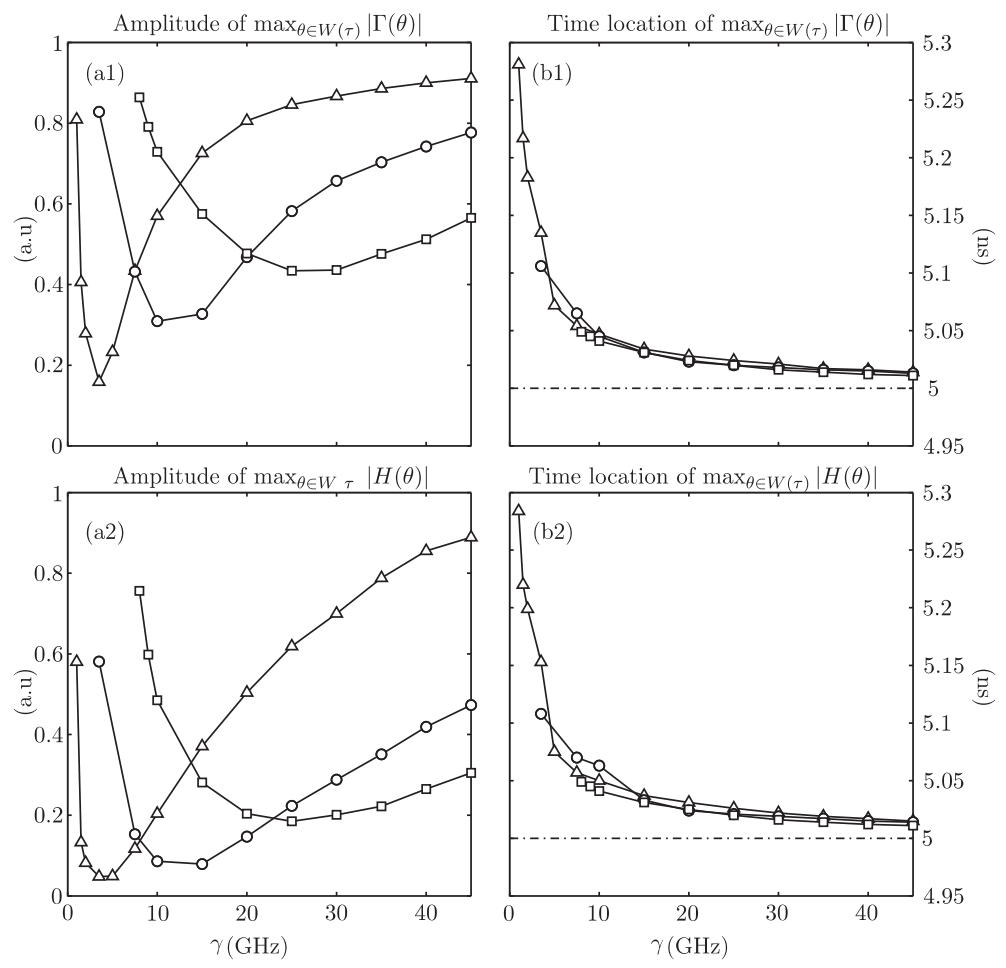

Fig. 2. Impact of the feedback variation and pumping factor on the amplitude and time location of the most significant peak in the vicinity $W(\tau)=$ [4.5ns, $5.5 \mathrm{~ns}]$ of the time delay $\tau=5 \mathrm{~ns}$. (a1)-(b1) gives respectively the amplitude and time location of $\max _{\theta \in W(\tau)}|\Gamma(\theta)|$ (a2)-(b2) gives respectively the amplitude and time location of $\max _{\theta \in W(\tau)}|H(\theta)|$. The solid lines with triangle $(\triangle)$, round (o), and square $(\square)$ markers stands for $p=1.05, p=1.26$ and $p=1.72$, respectively. These three different values of $p$, correspond to the following relaxation oscillation periods: $\tau_{R O}=0.75 \mathrm{~ns}, \tau_{R O}=0.33 \mathrm{~ns}$ and $\tau_{R O}=0.2 \mathrm{~ns}$, respectively. In sub-figures (b1)-(b2), the dotted-dashed lines give the time location of the time-delay $\tau$. 
delay signature will not be easily retrievable either in the ACF or the DMI.

Figure 3 illustrates this fact for $\tau=1.2 \mathrm{~ns}$ and keeping $\tau_{R O}=0.75 \mathrm{~ns}$.

At large feedback rate, the reduction of the time separation between $\tau$ and $\tau_{R O}$ does not produce a qualitative change of the time-delay identification at strong feedback rates. Both estimators still exhibit an easy-retrievable time-delay signature: a sharp peak close to the time-delay location [Fig. 3(b3)-(c3) and (b4)-(c4)]. Then, similar to Fig. 1, a diminution of the feedback rate, promotes the effect of $\tau_{R O}$. This leads to a time-delay signature hardly retrievable for sufficiently close values of $\tau$ and $\tau_{R O}$ [Fig. 3(b2)-(c2)], whereas it was still possible to retrieve it when the two timescales were sufficiently disparate [Fig. 1(b2)-(c2)]. We notice also that the ACF or DMI evolve from a pulse-like shape at high feedback rates to an oscillating shape at low feedback rate [Figs 3 (b1) and (c1)]. The transition between these two shapes is achieved for a feedback rate close to the one that leads to a minimum in the curves of figs. 2 (a1)-(a2), which corresponds to a particularly adapted situation to conceal the time-delay signature, since the two timescales equivalently drive the dynamics of the intensity time series. The exponentially-damped oscillations of the ACF mask the time-delay signature if $\tau$ and $\tau_{R O}$ are sufficiently close. Indeed, the peaks and valleys are temporally located at multiples of $\tau_{R O}$ and multiples of $\tau_{R O} / 2$, respectively, and no signature of the time delay remains, by contrast to the situation where the difference between $\tau_{R O}$ and $\tau$ was larger [Fig. 1(b1)-(c1)].

\section{TIME-DELAY IDENTIFICATION: A DYNAMICAL POINT OF VIEW}

The previous section has shown that the identification of the time-delay in the chaotic output of a laser with optical feedback strongly depends on the operational parameters of the ECSL: the feedback rate $\gamma$, the pumping current $p J_{t h}$, and the value of $\tau$ relatively to $\tau_{R O}$. At high feedback rates, the estimators always possess a predictable behavior: a pulse-like shape with a clear signature of $\tau$. However, when the feedback rate is weak, a large variety of behaviors has been reported and detailed in the previous section. These behaviors are largely dependent on the choice of $p$ and $\tau$ relatively to $\tau_{R O}$.

These first results suggest a closer analysis of the timescales involved in the early stage of the laser chaotic dynamics, at small feedback rates. Different dynamical scenarios are expected depending on the ratio between $\tau$ and $\tau_{R O}$, which then also lead to the different time-delay identification scenarios we reported in the previous section.

\section{A. Influence of the frequency generation on the time-delay signature}

1) Disparate timescales scenario: In this section we analyze the ECSL dynamics when the feedback rate $\gamma$ is taken as the bifurcation parameter, all the other parameters remaining identical to the ones of Fig. 1. Figure 4(a) shows a bifurcation diagram of the laser intensity. A quasi-periodic (QP) route to chaos is observed. A projection of the system trajectory in the phase plane $(N, E)$, the power spectrum, the $\mathrm{ACF}$ and the DMI, are plotted in Fig. 5 for specific values of the feedback rate. As we increase the feedback rate, the stationary solution of the ECSL is destabilized through a first Hopf bifurcation (H1) which induces a time-periodic dynamics [Fig. 5(a1)], with a frequency $f_{H 1}=1.32 \mathrm{GHz}$ almost equal to the relaxation-oscillation frequency $f_{R O}=1 / \tau_{R O}=1.33 \mathrm{GHz}$ [Fig. 5(b1)]. The intensity $I(t)$ being time-periodic, the ACF and the DMI exhibit an oscillating shape at the frequency of the limit cycle oscillation. In the ACF, the maximum correlation $\Gamma(\theta)=1$ and anti-correlation $\Gamma(\theta)=-1$ are reached at $\theta \approx k \tau_{R O}$ and $\theta \approx k \tau_{R O} / 2$, respectively. The DMI presents two series of peaks with different amplitudes: one located at $\theta \approx k \tau_{R O}$ and the other at $\theta \approx k \tau_{R O} / 2$.

Increasing the feedback rate, the limit cycle destabilizes to a quasiperiodic dynamics [Fig. 5(a2)]. The power spectrum shows the appearance of new frequencies [Fig. 5(b2)]. Amongst them, one strong frequency component appears separated from $f_{H 1}$ by $\Delta f=0.19 \mathrm{GHz}$ very close to the external-cavity frequency $f_{E C}=1 / \tau=0.2 \mathrm{GHz}$.

This new frequency component induces a slow undamped periodic modulation of both the ACF and DMI [Fig. 5(c2)(d2)]. Further increase of the feedback rate is accompanied by the appearance of numerous new frequencies [Fig. 5(b3)] that increase the attractor complexity [Fig. 5(a3)]. However, the strong frequency components are still located at the frequencies $f_{H 1}$ and $f_{H 1}-\Delta f$, which guarantees the persistence of a global order of the time series on a long time-extension even if on a short time-extension the complexity (disorder) is increased. As a consequence, the ACF and DMI show a stronger modulation, comparing to Fig. 5(c2)-(d2). This is due to the local increase of complexity, although periodically the ACF still attains both maximum correlation and anticorrelation [Fig. 5(c3) and (d3)]. For still larger feedback rate the laser exhibits fully developed chaos [Fig. 5(a4)]. The intensity time series progressively looses correlation as time increases, which corresponds to damp the modulated shape of the ACF and DMI [Fig. 5(c4) and (d4)]. Although the frequency content of the chaotic output is broad, the ACF and DMI functions still retain signatures of the first time-scales that appear in the bifurcation cascade: the undamped relaxationoscillation period $\tau_{H 1} \approx \tau_{R O}$ that appears after the first Hopf bifurcation and the time delay $\tau$ that appears when crossing a torus bifurcation to quasiperiodic dynamics. The undamped relaxation oscillations are responsible for the oscillatory shape of the ACF and the DMI functions, and then the time delay time-scale appears from a bifurcation to quasiperiodicity and slowly modulates the shapes of the ACF and DMI functions. The fast oscillations at the first Hopf bifurcation, close to RO frequency, perturb the identification of the time-delay at weak feedback rate, close to the onset of chaos. Only high feedback rates sufficiently weaken the relaxation-oscillation signature, namely short-time correlation, and offers a clear time-delay signature.

2) Close timescales scenario: Similarly, the influence of the bifurcation cascade is investigated for close values of the two timescales $\tau$ and $\tau_{R O}$. In this case, the analysis is performed using parameters identical to the ones in Fig. 3. The bifurcation 

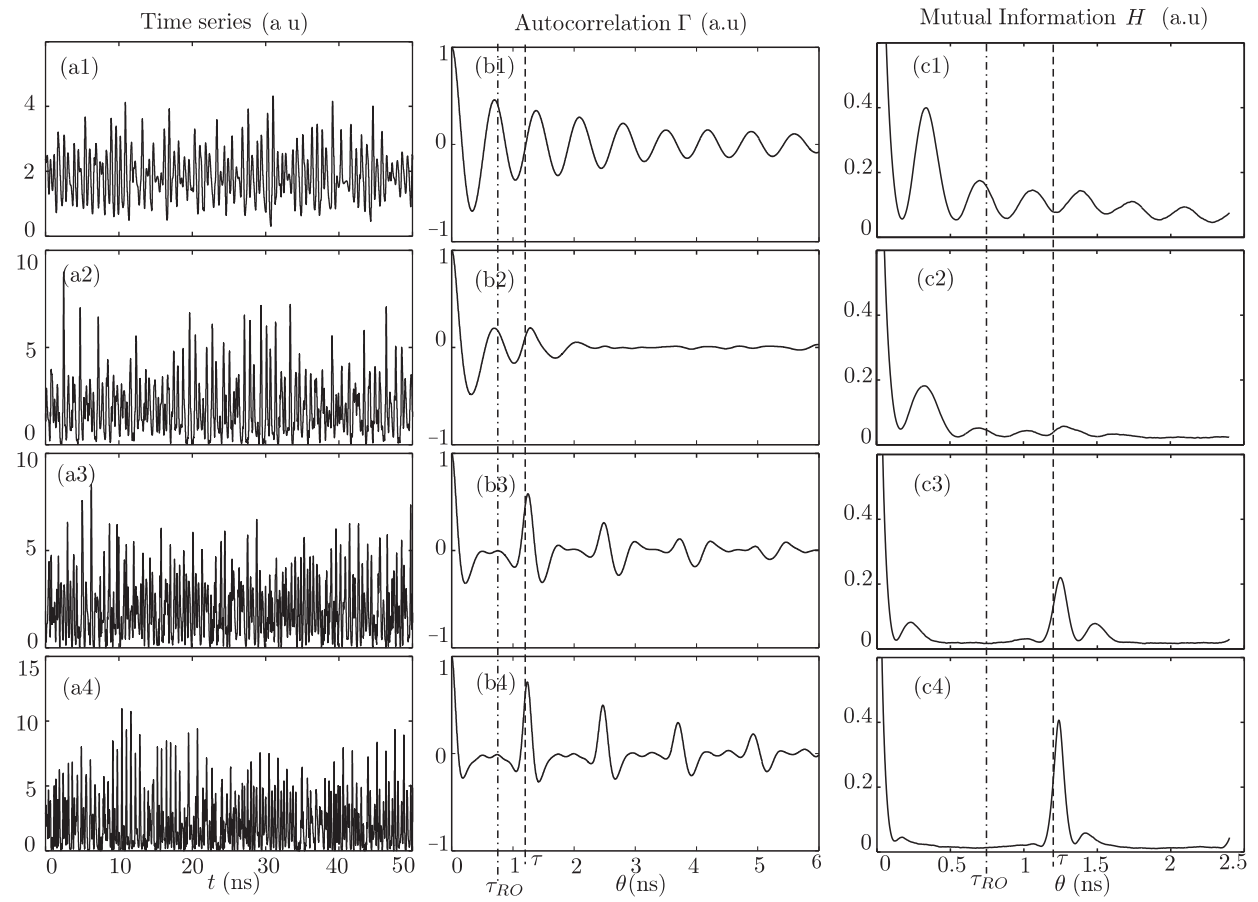

Fig. 3. ECSL intensity time series (1st column), autocorrelation function (2nd column) and mutual information (3rd column) for increasing value of the feedback rate $\gamma=2 \mathrm{GHz}$ (1st row), $\gamma=5 \mathrm{GHz}$ (2nd row), $\gamma=10 \mathrm{GHz}$ (3rd row), $\gamma=15 \mathrm{GHz}$ (4th row) with a time-delay value $\tau=1.2 \mathrm{~ns}$ and $\tau_{R O}=0.75 \mathrm{~ns}$. The vertical dotted-dashed and dashed lines give the time location of $\tau_{R O}$ and $\tau$, respectively.
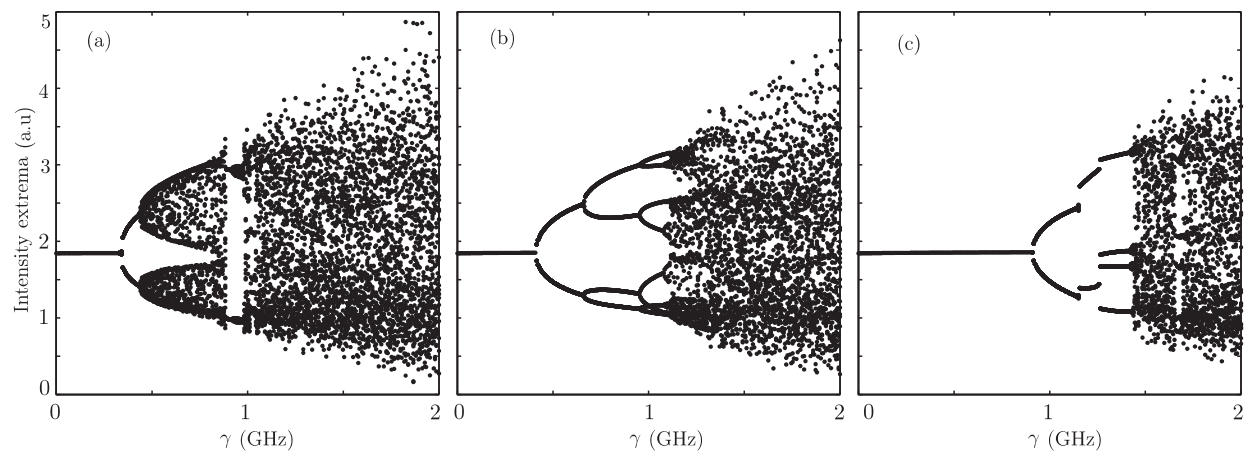

Fig. 4. Bifurcation diagrams with the feedback rate $\gamma$ taken as the bifurcation parameter. It shows various routes to chaos depending on the choice of $\tau$ and $\tau_{R O}$. (a) quasi-periodic (QP) route to chaos for $\tau=5 \mathrm{~ns}$ and $\tau_{R O}=0.75 \mathrm{~ns}$. (b) period-doubling (PD) route to chaos for $\tau=1.2 \mathrm{~ns}$ and $\tau_{R O}=0.75 \mathrm{~ns}$. (c) complex route to chaos for $\tau=0.85 \mathrm{~ns}$ and $\tau_{R O}=0.75 \mathrm{~ns}$

diagram in Fig. 4(b) shows a period-doubling (PD) route to chaos. We observe in this case that the frequencies generated by the ECSL nonlinear dynamics also significantly influence the shape of the estimators. However, unlike the previous case, the time-delay does not manifest itself in the early stages of the dynamics.

As previously, the progressive increase of feedback rate leads to a time-periodic dynamics with a frequency $f_{H 1}=$ $1.34 \mathrm{GHz}$ [Fig. 6(b1)] close to $f_{R O}$. This time-periodic dynamics induces an oscillating behavior of the ACF [Fig. 6(c1)] and DMI [Fig. 6(d1)] similar to Fig. 5(c1) and (d1). When further increasing the feedback rate, the laser exhibits a period doubling bifurcation, which yields a new frequency at $f_{H 1} / 2$ [Fig. 6 (b2)]. The coexistence of these two timescales modulates the shape of the estimators [Fig. 6(c2)-(d2)], making $2 \tau_{H 1}=2 / f_{H 1}$ and its multiples the time-locations of the strongest contributions in the time-delay estimators. Further increase of the feedback rate leads to the appearance of many frequencies in the ECSL power spectrum, and has two effects on the estimators: the global decrease of the amplitude of the oscillations of the ACF and DMI, and a stronger modulation [Fig. 6(b3)-(c3)] due to similar reasons as the ones in Fig. 5(c3)-(d3). The appearance of the chaotic regime produces exponential damping of both the ACF and DMI [Fig. 6(c4)(d4)]. Even in the chaotic regime the strongest frequency component is still found at the frequency $f_{H 1}=1.34 \mathrm{GHz}$. The ACF function exhibits fast oscillations at the period $\tau_{H 1}=1 / f_{H 1}$ but the correlation is lost on longer timeextension. In this identification scenario, and by contrast to the previous case, the time delay time-scale is not present in the early stages of the ECSL nonlinear dynamics. This prevents its clear signature to appear in the estimators at 

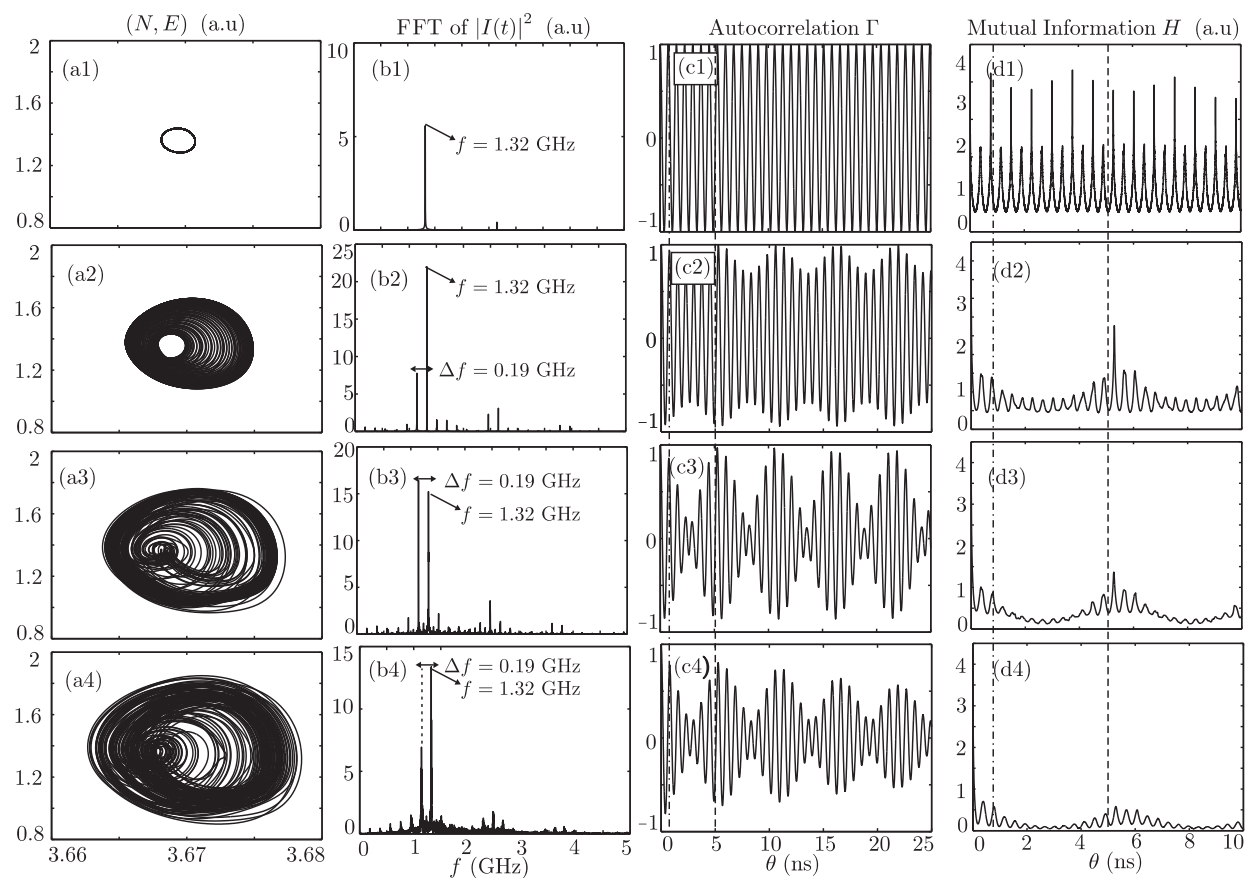

Fig. 5. Projection of the attractor in $(N, E)$ plane (1st column), Power spectrum (FFT of $|I(t)|^{2}$ ) (2nd column), autocorrelation function (3rd column) and mutual information (4th column) for increasing value of the feedback rate $\gamma=0.35 \mathrm{GHz}$ (1st row), $\gamma=0.55 \mathrm{GHz}$ ( $2 \mathrm{nd}$ row), $\gamma=0.85 \mathrm{GHz}(3 \mathrm{rd}$ row), $\gamma=1 \mathrm{GHz}$ (4th row) with a time-delay value $\tau=5 \mathrm{~ns}$ and $\tau_{R O}=0.75 \mathrm{~ns}$. The vertical dotted-dashed and dashed lines give the time location of $\tau_{R O}$ and $\tau$, respectively.

low feedback rates. Both the ACF and the DMI give only information concerning the value of the undamped relaxationoscillation period through an oscillating behavior.

In conclusion, we have shown that the cascade of bifurcations is responsible for the observed behavior of the estimator. The first frequency appearing in the ECSL leads to oscillations in both the ACF and DMI. Depending on the choice of the parameters, the time-delay signature may or may not appear early in the first stage of the ECSL nonlinear dynamics [Fig. 5(b2)]. This presence influences the security of the ECSL cryptosystem in terms of time-delay identification. However, the early appearance of $\tau$ is not the only aspect to consider: the ACF and DMI starting to oscillate at the period of the undamped relaxation oscillation $\tau_{H 1}$, it is of importance to also study the particular role of this frequency and its remaining presence as the ECSL undergoes its cascade of bifurcations.

\section{B. Influence of the first Hopf frequency and diversity of time- delay-identification scenarios}

The undamped relaxation-oscillation period $\tau_{H 1}$ emerging from $\mathrm{H} 1$ is the first timescale that appears in the ECSL. This makes $f_{H 1}=1 / \tau_{H 1} \approx f_{R O}$ play the role of a fundamental frequency of the ECSL. The presence of this fundamental frequency seems to be systematically responsible for the oscillating behavior of the estimators and can blur or even mask the time-delay signature [Figs. 1 and 3]. However, in this section, we will underline two important facts: first, the frequency $f_{H 1}$ can be significantly shifted from $f_{R O}$; second, the frequency emerging from $\mathrm{H} 1$ in the ECSL will not always play the role of the fundamental frequency responsible for the oscillating behavior of the ACF and DMI.
1) Frequency shift and influence on the time-delay identification: In the previous cases of Figs. 1 and $3, f_{H 1}$ was found to be extremely close to the value of $f_{R O}$. We have made in Fig. 7 a more systematic analysis of the first Hopf bifurcation frequency $f_{H 1}$ as a function of the time delay $\tau$. The evolution of $f_{H 1}$ with $\tau$ is not monotonic; it periodically oscillates around a value corresponding to $f_{R O}$ (dashed line) and the period of oscillation is close to $\tau_{R O}$. A similar conclusion has been obtained for other sets of parameters in previous ECSL studies [17]-[20]. This property can be used to increase the security of the ECSL. Indeed, the oscillating behavior of the ACF and DMI is characteristic of the presence of the undamped relaxation-oscillation timescale. Our previous choice of the parameters $\tau=1.2 \mathrm{~ns}$ or $\tau=5 \mathrm{~ns}$ and $\tau_{R O}=0.75 \mathrm{~ns}$ has led to values of $f_{H 1}$ both close to $f_{R O}$. Now if we choose a value of the time delay $\tau$ close to $\tau_{R O}$, the fundamental frequency at which the ECSL will oscillate will be significantly shifted from $f_{R O}$; when combined with a low feedback rate, we expect a damping oscillating behavior of both the ACF and DMI at a time-scale that corresponds neither to $\tau$ nor to $\tau_{R O}$, hence preventing a good time-delay identification.

2) Diversity of time-delay-identification scenarios: In the previously investigated cases, the shape of the estimator (ACF or DMI) unveils the influence of the first timescale appearing in the ECSL, namely the frequency $f_{H 1}$. However it appears that the frequency issued from the first Hopf bifurcation does not systematically play the role of the fundamental frequency that drives the shape of the ACF or DMI for larger feedback rates. The following example taking $\tau_{R O}=0.75 \mathrm{~ns}$ and $\tau=0.85$ ns illustrates this fact. Figure 4(c) shows the corre- 

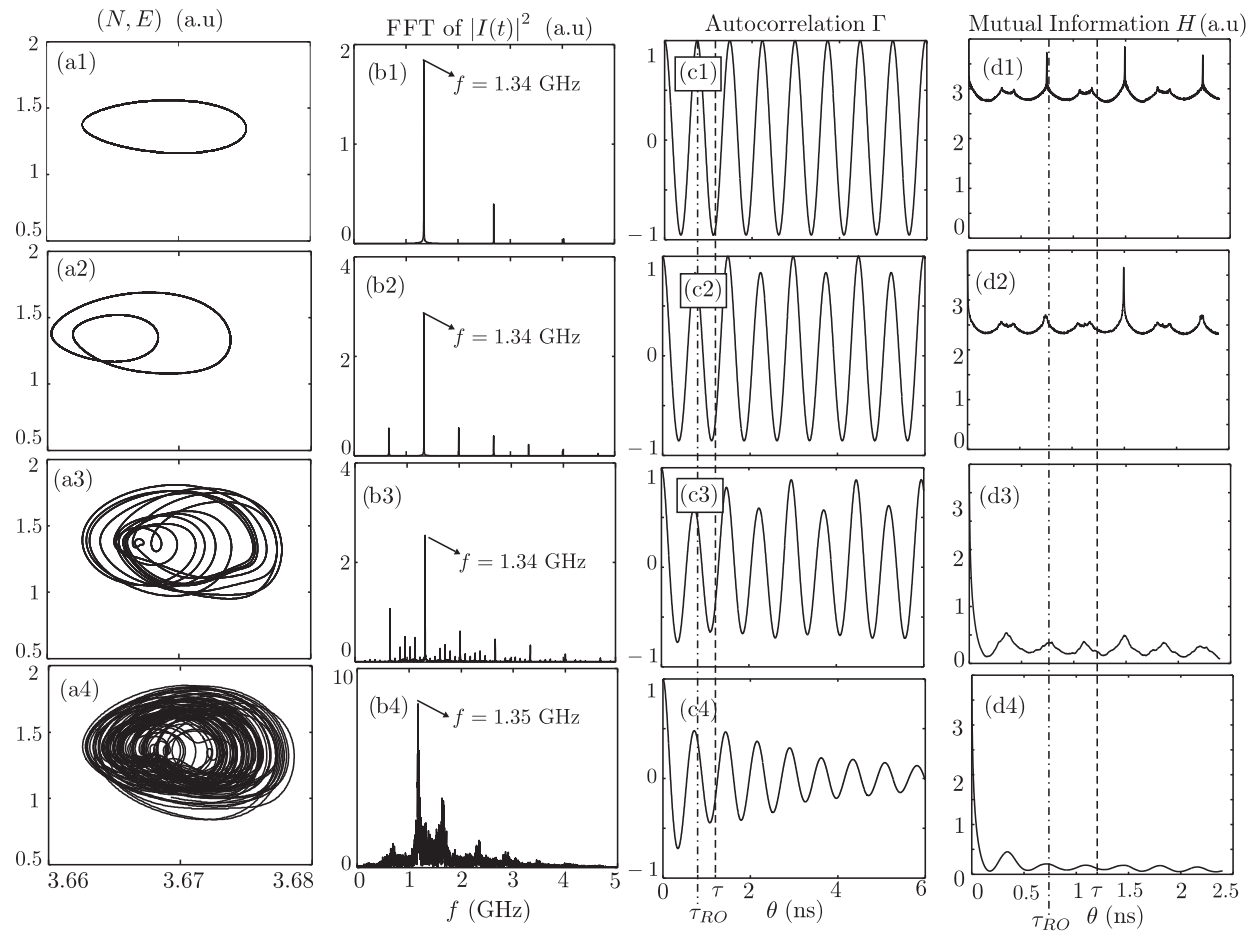

Fig. 6. Projection of the attractor in $(N, E)$ plane (1st column), power spectrum (FFT of $\left.|I(t)|^{2}\right)$ (2nd column), autocorrelation function (3rd column) and mutual information (4th column) for increasing values of the feedback rate: $\gamma=0.6 \mathrm{GHz}$ (1st row), $\gamma=0.8 \mathrm{GHz}(2 \mathrm{nd}$ row), $\gamma=1.2 \mathrm{GHz}(3 \mathrm{rd}$ row), $\gamma=1.5 \mathrm{GHz}$ (4th row) with a time-delay value $\tau=1.2 \mathrm{~ns}$ and $\tau_{R O}=0.75 \mathrm{~ns}$. The vertical dotted-dashed and dashed lines give the time location of $\tau_{R O}$ and $\tau$, respectively.

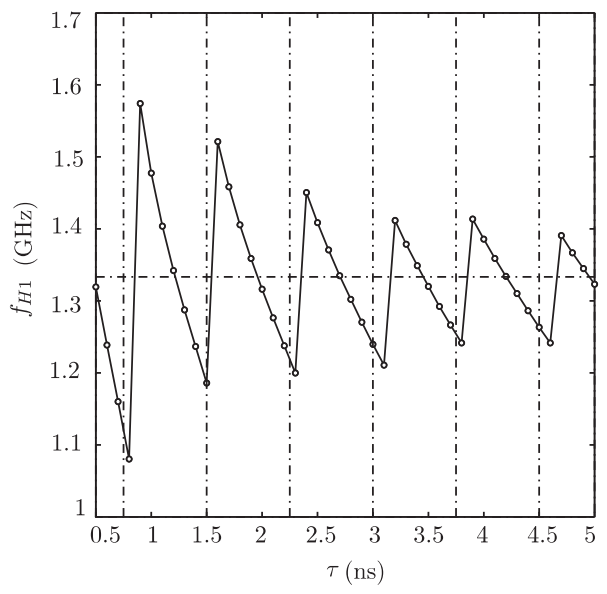

Fig. 7. Evolution of the first Hopf Frequency $f_{H 1}$ as a function of the time delay $\tau$, for $\tau_{R O}=0.75 \mathrm{~ns}$. The horizontal and vertical dotted-dashed lines represent respectively the relaxation oscillation frequency $f_{R O}=1 / \tau_{R O}$, and multiples of the relaxation-oscillation period $\tau_{R O}$.

sponding bifurcation diagram with a complex route to chaos. The first frequency $f_{H 1}=1.64 \mathrm{GHz}$ significantly shifted from $f_{R O}=1.33 \mathrm{GHz}$ [Fig. 8(b1)] induces an oscillating behavior of the ACF and DMI [Fig. 8(c1) and (d1)]. Successive increases of feedback rate first destabilize the limit cycle born from $H 1$ and lock the ECSL on a new limit cycle with a frequency (also shifted from $\left.f_{R O}\right) f=1.02 \mathrm{GHz}$ [Fig. 8(b2)], and second make the ECSL locks on a period-doubled limit cycle with fundamental frequency $f=0.88 \mathrm{GHz}$ [Fig. 8(b3)]. This induces new oscillating behaviors for ACF and DMI with fundamental frequencies largely shifted either from $f_{H 1}$ or $f_{R O}$ [Fig. 8(c2)-(c3)]. Finally, the system enters in a weakly developed chaotic regime that inherit the spectral contents of the last stable attractor [Fig. 8(b3)-(b4)]. This explains also the inherited shape of the ACF between Fig. 8(c3) and (c4). In Fig. 8(c4), the ACF exhibits a largely shifted peak issued from the fundamental frequency of the last stable limit cycle [Fig. 8(a3)] which can be considered as a possible timedelay signature. In conclusion, we have shown that the first Hopf frequency is not systematically responsible for the fast oscillating shape of the ACF or DMI. We have also illustrated, again, the key role of the other frequencies born from the cascade of bifurcation occurring in the ECSL: they shape in a non-trivial way the ACF and the DMI, and are responsible for blurring the time-delay signature. The resulting identification scenario is not completely similar to the previous ones in Figs. 5 and 6. This different identification scenarios take their origin in the diversity of routes to chaos existing in the ECSL, which influence directly on the laser time-series and shape the estimators accordingly.

\section{DISCUSSION}

In this section, we discuss the robustness of the time-delay concealment with respect to other identification techniques and to the influence of laser spontaneous emission noise and gain compression coefficient, as well as potential applications of our results for chaos-based communications. 

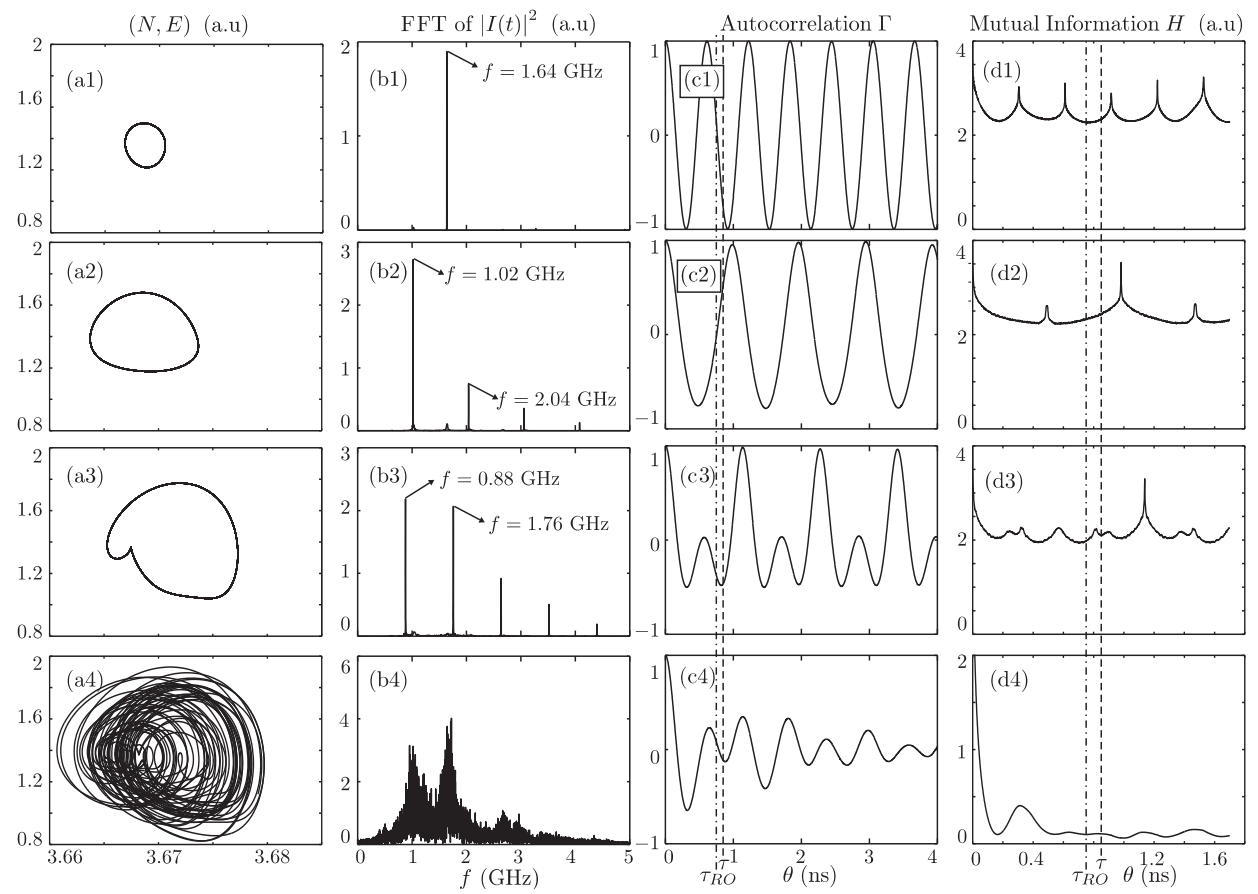

Fig. 8. Projection of the attractor in $(N, E)$ plane (1st column), power spectrum (FFT of $\left.|I(t)|^{2}\right)$ (2nd column), autocorrelation function (3rd column) and mutual information (4th column) for increasing values of the feedback rate: $\gamma=1 \mathrm{GHz}$ (1st row), $\gamma=1.2 \mathrm{GHz}$ (2nd row), $\gamma=1.4 \mathrm{GHz}$ (3rd row), $\gamma=1.6 \mathrm{GHz}$ (4th row) with a time-delay value $\tau=0.85 \mathrm{~ns}$ and $\tau_{R O}=0.75 \mathrm{~ns}$. The vertical dotted-dashed and dashed lines give the time location of $\tau_{R O}$ and $\tau$, respectively.

\section{A. Robustness of time-delay concealment to other identifica- tion techniques}

Numerous methods exist to identify the time-delay, besides the well known ACF and DMI. The most common ones are: the filling factor analysis [22], the local linear models (LLMs) [10], global nonlinear models such as those obtained with modular neural networks [23], and statistics of time-series extrema [24]. It is however interesting to analyse whether these time-delay-identification techniques would allow to retrieve any information on the time-delay value.

We present here a time-delay extraction based on modular neural networks (MNN). In order to mimic the structure of the equation driving the evolution of the light intensity $I(t)$, the MNN incorporates two modules, one for the non delayed part (ND) of $I(t)$ and a second one for its delayed part (D). A feed-forward neural network [23] is used for each of the modules. The first module is fed with input data: $x_{N D}=$ $\left[I\left(t-\tau_{e}\right), \ldots, I\left(t-m_{1} \tau_{e}\right)\right]$ and the second module is fed with $x_{D}=\left[I\left(t-\theta-m_{2} \tau_{e}\right), \ldots, I(t-\theta), \ldots, I\left(t-\theta+m_{2} \tau_{e}\right)\right]$. The parameter $\tau_{e}$ is the embedding time, $\theta$ is a time-delay candidate, $m_{1}$ is the number of inputs of the ND part, and $2 m_{2}+1$ is the number of required inputs for the delayed part [10]. The output of the neural network is

$$
x_{n n}=f\left(x_{N D}\right)+h\left(x_{D}\right),
$$

where $f$ and $h$ are nonlinear functions associated to ND and $D$ respectively. The forecast error of the MNN is defined by $\sigma(\theta)=\left\|I(t)-x_{n n}\right\|$, and is a function of $\theta$. If there exists a value $\theta_{0}$ such that $\sigma\left(\theta_{0}\right)$ is minimum, corresponding to a valley in the forecast-error curve, then $\theta_{0}$ will be considered a time- delay estimation. We apply the MNN technique to the intensity time series corresponding to the operational parameters $\tau=1$ $\mathrm{ns}, \tau_{R O}=0.75 \mathrm{~ns}$, and $\gamma=2 \mathrm{GHz}$. In previous sections, we have shown that such a set of parameter leads to a chaotic laser output from which it is not possible to retrieve the information on the time-delay using ACF or DMI. Figure 9 presents the results of a time-delay extraction based on a MNN composed of 6 neurons in the first layer and 3 neurons in the second layer for the delayed module and only one neuron for the non delayed module. The plot of the forecast error reveals only a minimum for values $\theta$ close to 0 , that corresponds to the linear correlation time [23]. We do not observe, however, any other minimum in the vicinity of the time delay $\tau$. As a consequence, the time delay cannot be inferred from the intensity time-series using MNN.

The other identification techniques described above have also been tested and do not give more insight about the timedelay value. However, it is interesting to note that the statistics of time-series extrema is among all methods the one that identifies the time delay of the system for the smallest value of the feedback rate $\gamma$.

In conclusion, we have shown that independently from the method of identification, if the operational parameters of the ECSL are carefully chosen, the time-delay signature remains hidden.

\section{B. Influence of internal parameters}

1) Influence of the spontaneous-emission noise: In our study, we have considered that the ECSL is fully deterministic; as a consequence, a description based on a deterministic delaydifferential equation was sufficient. However, in real lasers, 


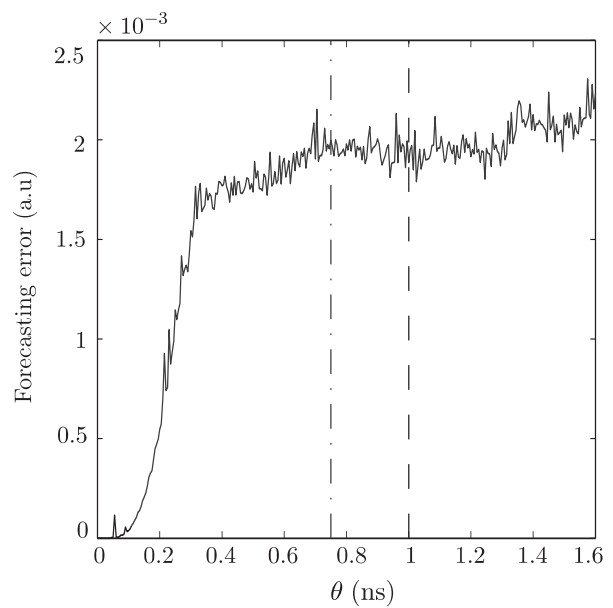

Fig. 9. Forecast error produced by the MNN on the intensity time series $I(t)$ generated with the following parameters: $\tau=1 \mathrm{~ns}, \tau_{R O}=0.75 \mathrm{~ns}$ and $\gamma=2 \mathrm{GHz}$. The identification time-step is $5 \mathrm{ps}$. The vertical dotted-dashed and dashed lines give the time location of $\tau_{R O}$ and $\tau$, respectively.

stochastic processes, such as spontaneous emission, occur in the gain medium. Such phenomena therefore require an adapted model. The influence of the spontaneous-emission noise is modelled by a Langevin force and a deterministic component added to the equation describing the time evolution of the complex electric field $E(t)$ [see Eq. (1)]. This additional stochastic part of the LK equations blurs the cascade of bifurcations that has proven to be directly responsible, in a fully deterministic approach, for the blurred time-delay signatures observed in both the ACF and DMI. Indeed, when noise is present at very weak feedback rates, its contribution in the ECSL dynamics is comparable to that of the delayed optical feedback term. The Langevin force $F_{L}(t)=\sqrt{2 \beta N(t)} \zeta(t)$ acts as a driving force for the dynamics that weakly excite the intrinsic nonlinearity of the ECSL. This stochastic excitation, only visible at low feedback rate, does not influence the time-delay identification: the signature is still blurred. For large values of feedback rates, the contribution of the noise is negligible in comparison with the delayed-feedback term. This implies that the time-delay signature remains identical to the one in Fig. 1(b4)-(c4). Figure 10 shows this result by comparing the evolution for increasing feedback rate strength of a model of ECSL with and without spontaneous-emission noise. In conclusion, the presence of noise in the equation does not affect the time-delay identification, and the results of section III remain true.

2) Influence of gain saturation: Gain saturation is phenomenologically introduced in the rate equations by considering an explicit intensity dependence of the gain. Its value is typically smaller or equal than $2.5 \times 10^{-23} \mathrm{~m}^{3}$. It has been reported elsewhere that the saturation gain has a stabilizing effect on the ECSL dynamics: the progressive increase of gain saturation leads to an increasing feedback-strength threshold above which the transition to chaotic regime occurs [25].

This stabilizing effect of gain saturation is due to the damping effect of the intrinsic nonlinear part of rate equation (1). As a consequence, reducing the value of $\varepsilon$ will increase, in the ECSL dynamics, the driving action of $G_{N,|E|} E(t)$ relatively to the one of $\gamma e^{i \omega_{0} \tau} E(t-\tau)$, blurring the timedelay signature compared to fast timescales emerging from the interplay of the laser intrinsic nonlinearities and the optical feedback. From a quantitative point of view, a decrease of the gain saturation has two simultaneous manifestations: first, the amplitude of the extremum located around the timedelay value decreases, second fast oscillations appearing in the vicinity of the time delay and leads to a blurred timedelay signature. These two combined effects also allow for an increase of the time separation between $\tau$ and $\tau_{R O}$ that maintains the time-delay concealment. It also allows for the use of higher values of pumping current $p J_{t h}$. Figure 11 presents these results: three different identification scenarios based on the ACF are considered for various choices of $\tau, \tau_{R O}$ and $\gamma$ for a value of the gain saturation $\varepsilon$ that progressively increases. Interestingly, the first row shows, at a fixed feedback rate, that for a large time separation between $\tau$ and $\tau_{R O}$, where it may be expected to result in a blurred signature [Fig. 11(d4)], a progressive tendency to the disappearance of the time-delay signature is observed [Fig. 11(d1)]. These results hold for the various parameters used in Fig. 11. In conclusion, small values of the gain saturation favors the masking of the time-delay by emphasis the contribution of the intrinsic nonlinearity in the intensity time-series. They furthermore allow for extending the range in which the timedelay identification is difficult both in terms of feedback rate and separation of time-scales.

\section{Application to chaos-based communications}

The security in terms of time-delay identification appears as a requirement for the security of chaos-based communication setups involving physical devices such as ECSLs. However, it needs to be compatible with a sufficient chaos complexity as well as good synchronization, to ensure secure communications at low bit-error rates (BER). Indeed, it is important to investigate if the signal generated, with a good time-delay concealment, using our set of parameters, can possibly lead to secure transmission that can be recovered at the receiving end using chaos synchronization. Typically, the ECSL unidirectional coupling is used to form a chaotic communication scheme [3]. Two types of synchronization exist in such a scheme: the so-called injection-locking synchronization (generalized synchronization) and complete synchronization [26]. The first regime occurs when the master and slave ECSLs have identical optical feedback strengths $\left(\gamma_{m}=\gamma_{s}\right)$, whereas the second regime is achieved for a master optical feedback equal to the sum of the slave optical feedback with the coupling strength $\left(\gamma_{m}=\gamma_{s}+\gamma_{c}\right)$, providing the condition that $\gamma_{c}>\gamma_{s}$. The measurement of the quality of synchronization is based on the cross-correlation function defined by

$$
\Gamma_{X_{m}, X_{s}}(\theta)=\frac{\left\langle\left(X_{m}(t)-\mu_{X_{m}}\right)\left(X_{s}(t+\theta)-\mu_{X_{s}}\right)\right\rangle}{\sigma_{X_{m}} \sigma_{X_{s}}},
$$

with $\mu_{X_{m, s}}=\left\langle X_{m, s}\right\rangle$ and $\sigma_{X_{m, s}}=\left\langle\left(X_{m, s}-\mu_{X_{m, s}}\right)\right\rangle^{1 / 2}$. With the set of parameters of Fig. 3(b2)-(c2), a good timedelay concealment is achieved, but it leads to a poor quality of 


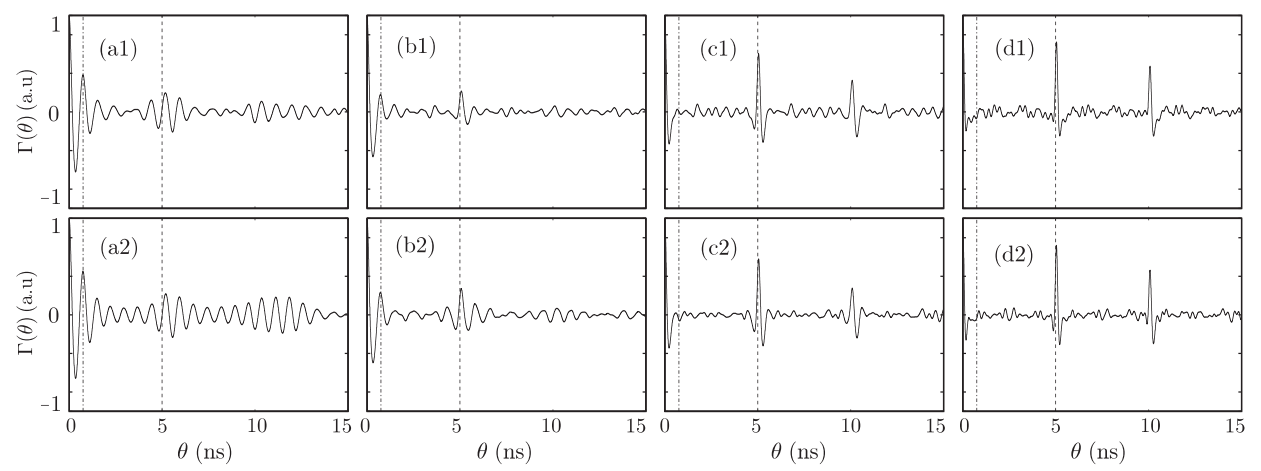

Fig. 10. Influence of the rate of spontaneous emission $\beta$ on the time delay identification. The first row corresponds to the case with the spontaneous emission noise $\beta=10^{3} \mathrm{~s}^{-1}$, the second row corresponds to the case $\beta=0 \mathrm{~s}^{-1}$. Each column is associated to a given feedback rate. From left to right, the feedback rates are: $\gamma=2.5 \mathrm{GHz}, \gamma=5 \mathrm{GHz}, \gamma=10 \mathrm{GHz}$ and $\gamma=15 \mathrm{GHz}$. The time delay and the relaxation period are equal to $\tau=5 \mathrm{~ns}$ and $\tau_{R O}=0.75 \mathrm{~ns}$. They are represented by the dotted and dotted-dashed lines, respectively.
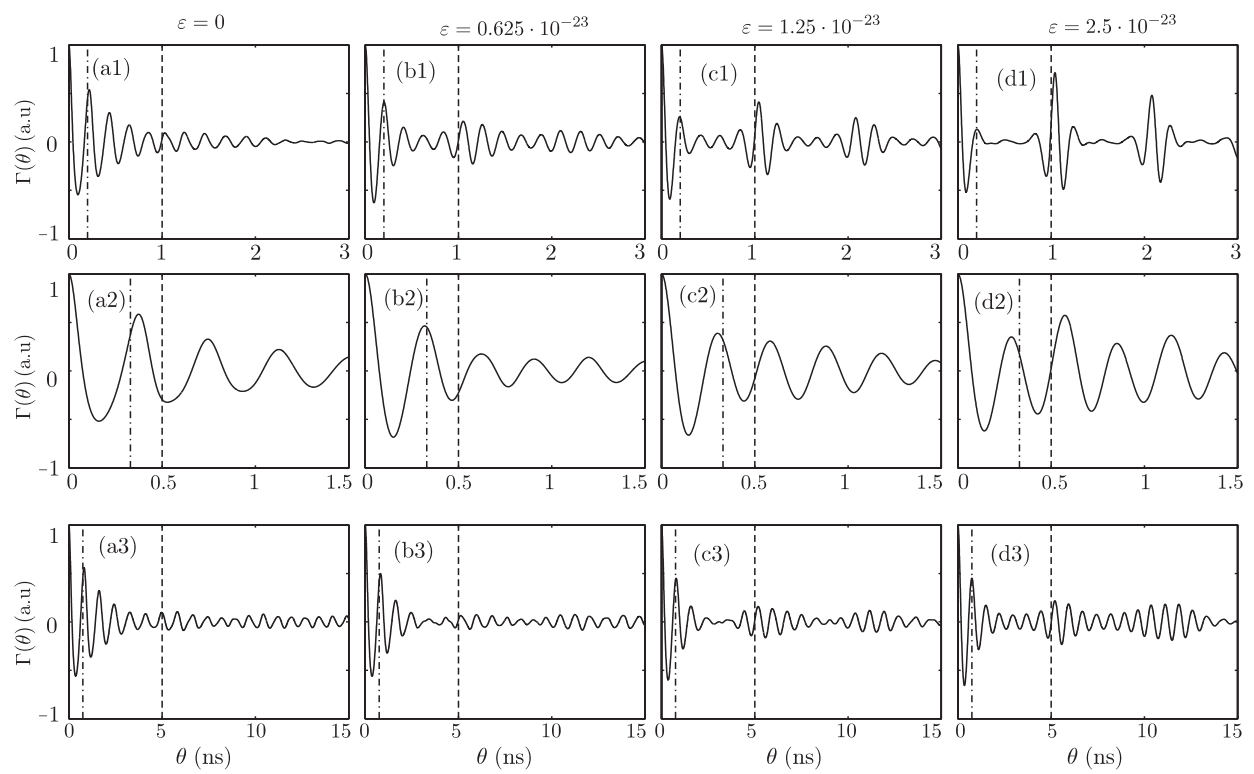

Fig. 11. Influence of the gain saturation $\varepsilon$ on the time delay signature for three different cases of difficult identification. The first row corresponds to $\tau=1$ ns, $\tau_{R O}=0.2 \mathrm{~ns}, \gamma=10 \mathrm{GHz}$, the second row to $\tau=0.5 \mathrm{~ns}, \tau_{R O}=0.33 \mathrm{~ns}, \gamma=5 \mathrm{GHz}$ and the third row to $\tau=5 \mathrm{~ns}, \tau_{R O}=0.75 \mathrm{~ns}, \gamma=2.5 \mathrm{GHz}$. The vertical dotted and dotted-dashed lines represent the time delay and the relaxation oscillation period, respectively. Each column corresponds to a different value of the saturation gain, as indicated on the figure.

anticipating synchronization with $\max _{\theta}\left|\Gamma_{X_{m}, X_{s}}(\theta)\right| \approx 0.2$. This result was expected because of the use of weak feedback rate in the master ECSL. In order to achieve complete synchronization the coupling strength must be equal to the feedback rate and must therefore take a value less than $5 \mathrm{GHz}$. In the case of injection-locking synchronization, by contrast, the coupling strength $\gamma_{c}$ is no longer limited by the value of the master optical feedback $\gamma_{m}$. Numerical simulations show that as soon as the coupling strength satisfies $\gamma_{c} \geq 25 \mathrm{GHz}$ we achieve very good synchronization: $\max _{\theta}\left|\Gamma_{X_{m}, X_{s}}(\theta)\right| \geq$ 0.95 . Hence, injection-locking synchronization can be used to transmit information securely, when the parameters are taken to ensure time-delay concealment, whereas complete synchronization is not adapted.

\section{CONCLUSION}

In this paper, we have analyzed the security of an ECSL in terms of time-delay identification using standard time-delay estimators such as the autocorrelation function (ACF) and the delayed mutual information (DMI). The key role of the feedback rate $\gamma$ and the pumping factor, as well as the choice of the time-delay $(\tau)$ value in comparison with the relaxationoscillation period $\tau_{R O}$, has been underlined. It appears that the difficult identification scenarios occur for relatively weak feedback rates and weak pumping factors with close values of the two timescales $\tau$ and $\tau_{R O}$. These difficult identifications find their origin in the specific nonlinear dynamics and timescales appearing in the ECSL in its bifurcation cascade leading to chaos. Indeed, at weak feedback rates the chaos keeps reminiscence of the time-scales involved in the early stage of the laser dynamics, such as the undamped relaxation oscillation time-scale and possibly period-doubling and quasiperiodic dynamics. The time-delay estimators then exhibit complex modulated shapes showing these different laser dynamics timescales. When $\tau$ and $\tau_{R O}$ are close to each other either, the true value $\tau$ is efficiently concealed thanks to a shift of the first 
Hopf frequency $f_{H 1}$ with respect to the relaxation oscillation frequency, such that the laser output at the Hopf frequency starts pulsating at a frequency which is neither close to $\tau$ nor to $\tau_{R O}$. The impact of additional laser internal parameters is also investigated. It appears that the decrease in gain saturation coefficient allows to use more distant values of $\tau$ and $\tau_{R O}$ as well as to increase the pumping factor $p$ while maintaining time-delay concealment. The robustness of our results has been checked with other signal processing techniques such as neural networks and filling-factor methods. We expect our results to be of interest for a proper design of a laser chaotic emitter that would allow for the best concealment of its system parameters, hence also improving security in laser chaos-based communications.

\section{ACKNOWLEDGMENT}

The authors would like to thank L. Pesquera for useful discussions and suggestions. The authors acknowledge the support of Conseil Régional de Lorraine and Fondation Supélec. The work of D. S. Citrin was supported in part by the National Science Foundation by grant ECCS 0523923.

\section{REFERENCES}

[1] D. Lenstra, B. H. Verbeek, and J.D. Boef, "Coherence collapse in single mode semiconductor lasers due to optical feedback", IEEE J. Quantum Electron., vol. 21, pp. 674-679, 1985.

[2] C. Risch and V. Voumard, "Self pulsation in the output intensity and spectrum of GaAs-AlGaAs cw diode lasers coupled to a frequency selective external optical feedback", J. Appl. Phys., vol. 48, pp. 20832085, 1977

[3] C.R. Mirasso, P. Colet, and P. Garcia-Fernandez, "Synchronization of chaotic semiconductor lasers: Application to encoded communications", IEEE Phot.Tech. Lett., vol. 8, pp. 299-301, 1996.

[4] R. Vicente, et al., "Analysis and characterization of the hyperchaos generated by a semiconductor laser subject to a delayed feedback loop", IEEE J. Quantum Electron., vol. 41, no. 4, pp. 541-548, 2005.

[5] K. Ikeda and K. Matsumoto, "High-dimensional chaotic behavior of systems with time-delayed feedback", Physica D, vol. 29, pp. 223-235, 1987.

[6] P. M. Alsing, et al., "Encoding and decoding messages with chaotic lasers", Phys. Rev. E, vol. 56, pp. 6302-6310, 1997.

[7] J.-P. Goedgebuer, L. Larger, and H. Porte, "Optical cryptosystem based on synchronization of hyperchaos generated by a delayed feedback tunable laser diode", Phys. Rev. Lett., vol. 80, pp. 2249-2252, 1998.

[8] A. Argyris, et al., "Chaos-based communications at high bit rates using commercial fibre-optic links", Nature, vol. 437, pp. 343, 2005.

[9] X. Li, W. Pan, B. Luo, and D. Ma, "Mismatch robustness and security of chaotic optical communications based on injection-locking chaos synchronization”, IEEE J. Quantum Electron., vol. 42, no. 9,pp. 953-960, 2006.

[10] R. Hegger, M. J. Bünner, H. Kantz, and A. Giaquinta, "Identifying and modeling delay feedback systems", Phys. Rev. Lett., vol. 81, no. 3, pp. 558-561, 1998.

[11] M. J. Bünner, A. Kittel, J. Parisi, I. Fischer, and W. Elsässer, "Estimation of delay times from a delayed optical feedback laser experiment", Europhys. Lett. , vol. 42, pp. 353-358, 1998.

[12] M. W. Lee, P. Rees, K. A. Shore, S. Ortin, L. Pesquera, and A. Valle, "Dynamical characterisation of laser diode subject to double optical feedback for chaotic optical communications", IEE Proc. Optoelectron., vol. 152, no. 2, pp. 97-102, 2005.

[13] D. Rontani, A. Locquet, M. Sciamanna and D. S. Citrin, "Loss of timedelay signature in the chaotic output of a semiconductor laser", Opt. Lett., vol. 32, no. 20, pp. 2960-2962, 2007.

[14] B. Dorizzi, B. Grammaticos, M. LeBerre, Y. Pomeau, E. Ressayre, A. Tallet, "Statistics and dimension of chaos in differential delay systems", Phys. Rev. A, vol. 35, pp. 328-339, 1987.
[15] V.S Udaltsov, L. Larger, J.P Goedgebuer, A. Locquet, and D. S Citrin, "Security of chaos-based communication system ruled by delay differential equation. Recovery of time-delay", J. Opt. Technol., vol. 72, no.5, pp. 373-377, 2005

[16] R. Lang and K. Kobayashi, "External optical feedback effects on semiconductor injection lasers properties" IEEE J. Quantum Electron., vol. 16 , pp. 347-355, 1980.

[17] A. Murakami and J. Ohtsubo, "Stability analysis of semiconductor laser with phase-conjgate feedback", IEEE J. Quantum Electron., vol. 33, no. 10, pp. 1825-1831, 1997.

[18] A. Murakami and J. Ohtsubo, "Dynamics and linear stability analysis in semiconductor lasers with phase-conjugate feedback", IEEE J. Quantum Electron., vol. 34, no. 10, pp. 1979-1986, 1998.

[19] B. Tromborg, J. H. Osmundsen, and H. Olesen, "Stability analysis for a semiconductor laser in an external cavity", IEEE J. Quantum Electron., vol. 20, no. 9, pp. 1023-1032, 1984.

[20] C. Masoller, "Effect of the external cavity length in the dynamics of a semiconductor laser with optical feedback", Opt. Comm., vol. 128, pp. 363-376, 1996

[21] T. M. Cover and J. A. Thomas, "Elements of information theory",John Wiley \& Sons, New York, USA, 1991.

[22] M. J. Bünner, Th. Meyer, A. Kittel, and J. Parisi, "Recovery of the timeevolution equation of time-delay systems from time series", Phys. Rev. E, vol. 56, pp. 5083-5089, 1997.

[23] S. Ortin, J.M. Gutierrez, L. Pesquera, H. Vasquez, "Nonlinear dynamics extraction for time-delay systems using modular neural networks synchronization and prediction", Physica A, vol. 351, pp. 133-141, 2005.

[24] B.P. Bezruchko, A.S. Karavaev, V.I. Ponomarenko, M.D. Prokhorov, "Reconstruction of time-delay systems from chaotic time series", Phys. Rev. E, vol. 64, pp. 056216, 2001.

[25] C. Masoller, "Comparison of the Effects of Nonlinear Gain and Weak Optical Feedback on the Dynamics of Semiconductor Lasers", J. Quantum Electron., vol. 33, no. 5, pp. 804-814, 1997.

[26] A. Locquet, C. Masoller, and C.R. Mirasso Blondel, "Synchronization regimes of optical-feedback-induced chaos in unidirectionally coupled semiconductor lasers", Phys. Rev. E, vol. 65, pp. 056205, 2002.

[27] V. Ahlers, U. Parlitz, and W. Lauterborn, "Hyperchaotic dynamics and synchronization of external-cavity semiconductor lasers", Phys. Rev. E, vol. 58, pp. 7208-7213, 1998.

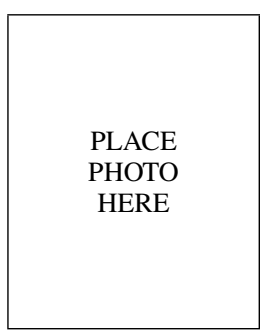

Damien Rontani received the M.S. degree in electrical and computer engineering both from the Ecole Supérieure d'Electricité (Supélec), Metz, France, and the Georgia Institute of Technology (Georgia Tech), Atlanta, USA in 2005 . He is currently working toward the Ph.D. degree jointly at Supélec and Georgia-Tech. His research interests include nonlinear dynamics of semiconductor lasers, security analysis of time-delay systems, chaos synchronization and cryptography.

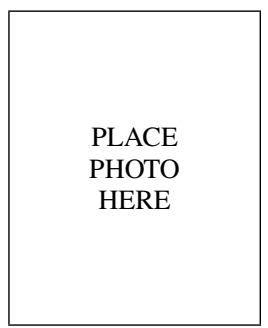

Alexandre Locquet (M'99) received the M.S. degree in electrical engineering from Facult Polytechnique de Mons (Belgium), in 2000, the Ph.D. degree in engineering science from Universit de FrancheComt (France), in 2004, and the Ph.D. degree in electrical and computer engineering from the Georgia Institute of Technology in 2005. His doctoral work was related to optical chaos-based communications. He is currently a postdoctoral researcher at the Unit Mixte Internationale Georgia Tech-CNRS, Metz, France. His interests are in physical-layer security, semiconductor laser dynamics, and time series analysis. 


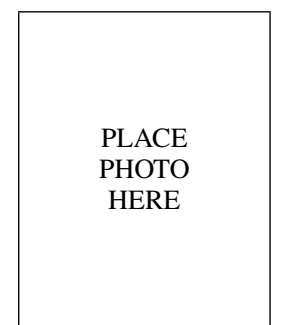

Marc Sciamanna graduated in electrical engineering from the Facult Polytechnique de Mons, Belgium, in 2000. He received the Ph.D. degree from Facult Polytechnique de Mons, as a Research Fellow of the "Fonds National de la Recherche Scientifique" (FNRS). His Ph.D. dissertation was entitled

"Nonlinear dynamics and polarization properties of externally driven semiconductor lasers." Currently, he is a permanent Researcher, Lecturer and faculty staff member of SUPELEC (Ecole Suprieure dElectricit), Metz, France, and LMOPS CNRS UMR-7132

Laboratory, Metz, France. His research interests include the physics and nonlinear dynamics of semiconductor lasers, the polarization properties of VCSELs, the study of dynamical instabilities related to optical feedback, optical injection, or large current modulation, synchronization and chaotic encryption using laser diodes. He is author of about 50 research papers in international journals and conference proceedings. He has given several invited talks in international conferences and has worked as a scientific program committee member in SPIE Photonics Europe 2006. He organized and cochaired the PHASE international workshop (PHysics and Applications of SEmiconductor LASERs), Supélec, Metz, France, in 2005; and acted as a Guest Editor of a special issue of Optical and Quantum Electronics related to the PHASE workshop. Dr. Sciamanna acted as a Board Member of the IEEE/LEOS Benelux Chapter, co-founded the IEEE/LEOS Benelux Student Chapter and co-chaired the IEEE/LEOS Workshop on Low-Cost Photonics (Mons, Belgium, June 13, 2003). He is Vice-Chairman of working group 2 "Physics of Devices" in European Action COST 288. He has been awarded with the Prize for Best Engineer in Electricity from Facult Polytechnique de Mons (2000), the OSA-Newfocus student travel grant award (2002), the IEEE/LEOS Graduate Student Fellowship Award (2002), and the SPIE FMADE Scholarship Award in Optical Engineering (2003).

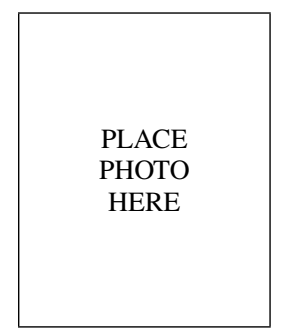

David S. Citrin (M'93-SM'03) received the B.A degree is in physics from Williams College, Williamstown, MA, in 1985, the M.S. and Ph.D. degrees in physics from the University of Illinois at Urbana-Champaign, in 1987 and 1991, respectively, where he worked on the optical properties of quantum wires. After receiving the Ph.D. degree, he was a Postdoctoral Research Fellow at the Max Planck Institute for Solid State Research, Stuttgart, Germany (19921993), where he worked on exciton radiative decay in low-dimensional semiconductor structures. From 1993 to 1995, he was Center Fellow at the Center for Ultrafast Optical Science at the University of Michigan, Ann Arbor, where his work addressed ultrafast phenomena in quantum wells. He was an Assistant Professor of Physics at Washington State University from 1995 to 2001, at which time he joined the faculty at the Georgia Institute of Technology, Atlanta, where he is currently a Professor in the School of Electrical and Computer Engineering. His interests are in ultrahigh-speed devices, nanoscale engineering, and terahertz technology. The present focus of his group is the identification and evaluation of novel nonlinear and ultrafast optical processes in various materials and nanostructures for applications in optoelectronics and photonics. His group also works on photonic crystals and on terahertz sources and sensors. Dr. Citrin has served as an Associate Editor for the IEEE JOURNAL OF QUANTUM ELECTRONICS.

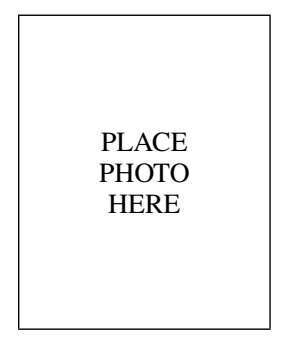

Silvia Ortin Silvia Ortin graduated in electrical engineering from the University of Cantabria, Spain, in 2002. She is currently working toward the Ph.D. degree at the Institute of Physics of Cantabria (IFCA). Her research interests include optical chaos communications, chaotic time series analysis and nonlinear dynamics. 